\title{
Power allocation optimization for fading channels in cognitive radio networks
}

\author{
Fareduddin Ahmed $\mathrm{J} \mathrm{S}^{\mathbf{1}^{*}}$ and Rohitha Ujjinimatad ${ }^{2}$ \\ Research Scholar, Department of Electrical and Electronics Engineering, VTU, Belgaum, Karnataka, India ${ }^{1}$ \\ Professor and HOD, Department of Electrical and Electronics Engineering, VTU, Belgaum, Karnataka, India ${ }^{2}$
}

Received: 12-November-2018; Revised: 05-February-2019; Accepted: 11-February-2019

C2019 Fareduddin Ahmed J S and Rohitha Ujjinimatad. This is an open access article distributed under the Creative Commons Attribution (CC BY) License, which permits unrestricted use, distribution, and reproduction in any medium, provided the original work is properly cited.

\begin{abstract}
The radio resource allocation in cognitive radio networks is an essential problem where the secondary users (SUs) use the spectrum allocated to primary users (PUs) without causing much interference to PUs. This paper studies optimal power and bandwidth allocation in a cognitive radio network under Rician and Nakagami-m fading channels. The performance metric for the network used is the ergodic-capacity of all the SUs. Further, this paper investigates the optimal power allocation schemes to achieve the primary capacity bounds of a secondary network with Rician and Nakagami-m fading channels. Specifically, the ergodic-capacity is considered. The methodology used involves deriving closed-form results for both Rician and Nakagami-m scenarios. Besides, the peak/average transmit-power constraints at the SUs and the peak/average interference power constraint imposed by the PU. The equations of optimal power allocations are also formulated under peak-power and peak-interference constraints. Further, the analysis is done for a network of SUs. Simulation results depicted with figures and tables show that the optimal power and bandwidth allocation for Rician and Nakagami-m fading channels. The investigation and analysis on optimal power and bandwidth allocation can be used for future reference of resource allocation in the cognitive radio networks over Rician and Nakagami-m fading channels.
\end{abstract}

\section{Keywords}

Cognitive radio, Spectrum allocation, Bandwidth allocation, Rician fading, Nakagami fading.

\section{Introduction}

The radio spectrum is turning out to be scarcer with the growing number of spectrum-hungry applications, then again, the report from the federal communications commission uncovered that most of the licensed spectrum is severely underutilized [1]. The difficulty between spectrum scarcity and spectrum underutilization has been managed in cognitive radio networks (CRNs) [2, 3]. Cognitive radio permits unlicensed users or secondary users (SUs) to access licensed spectrum to such an extent that the induced interference to the licensed users or primary users (PUs) is within the acceptable level. In cognitive radio, the SU senses for idle PU bands, and if found, SU may utilize these bands for its own data transmissions. The SU, however, performs spectrum sensing (SS) while transmitting its own to check the return of PU, and if PU has reappeared, SU has to empty the band immediately to maintain a strategic distance from harmful interferences.

*Author for correspondence

124
Various works have as of late contemplated data theoretic limits for radio resource allocation in CR. The development of maximum ergodic-capacity for the SU to target the optimal power allocation subject to the average-interference-power (AIP) and peakinterference-power (PIP) constraint set by a PU have been shown [4]. It is investigated that the SU outagecapacity, ergodic-capacity, and minimum-ratecapacity and developed the optimal power allocation for the same under both the AIP and PIP constraints from a PU [5]. The ergodic capacity, delay-limited capacity, and outage capacity of an SU are studied in [6]. The literature stated above [3-6], the study shows the system having only one SU and only in Rayleigh fading whereas, we consider a network of SUs and two fading channels. Even though fading channels have been extensively studied in the current literature [7-10]. They fail to address the issues of resource allocation on these fading channels. The study in [11], shows the two-way cognitive radio systems and addressed power allocation issues. However, recent investigation into [12] shows that the cognitive system with multiple SUs with multiple access channel models, where the peak power 
assignment is derived to attain the full sum-ergodiccapacity of the SUs for numerous power constraints. In [13], the author proposed a framework to control the selection of channels and assessed the framework execution of such a CRN. Some of the equations derived from the above stated works [11-13] have been used here and apply the considered channel conditions. The study in [14], shows that Nakagami$\mathrm{m}$ provides better bit error rate performance than Rician. The study in [15] shows that the capacity of Nakagami-m fading channels drastically increases compared to other Rayleigh, Rician and Weibull fading channels. The study in [16], shows the SS uses different methods with known and unknown noise levels. The study carried out for multilevel sensing and power allocation in Rayleigh fading channel have been discussed in $[17,18]$.

\subsection{Rician fading}

In realistic situations, the movement of the mobile device often results in the mobile to switch from the Line-Of Sight (LOS) path to a Non-Line-Of-Sight (NLOS) path and vice versa. Rician fading channel is a distinctive communication channel model in wireless and mobile systems, such as space, satellite, and naval communication, etc. In certain situations, the LOS component of the signal becomes very feeble. This happens when K-factor of the Rician fading channel is low. Only a few studies can be found on the Rician fading channel with respect to optimum power and bandwidth assignment in CRNs. It is worthwhile to investigate the performance of the sum ergodic capacity with respect to peak transmit power and interference in the Rician fading channel.

\subsection{Nakagami-m fading}

The Nakagami-m channel model was proposed by Nakagami in the early 1940s to depict fast fading in high-frequency channels. This channel model was selected as it is appropriate for experiential data and it is also handy, as it is more flexible and accurate in tallying some empirical data than other fading models. This channel model is most suitable for certain data signals used in metropolitan radio multipath-channels. Here $\mathrm{m}$ is the Shape factor of the Nakagami channel model. The various conditions from Rayleigh to Rician can be modeled by the Nakagami distribution; hence it is frequently used in terrestrial communication and satellite communication as a fading model. These interesting characteristics are the reasons for the extensive use of the Nakagami fading model to theoretical and empirical research in radio communication.

\section{Materials and methods}

\subsection{System model}

The cognitive radio system assumed (Figure 1) consists of one or more PUs and N SUs with a relay having multiple sensors. The PUs and the SUs operate in the same spectrum with bandwidth $\mathrm{W}$ for their transmissions. Let $h_{i}$ and $g_{i}$ denotes the channel gain between secondary transmitter and secondary receiver $\left(\mathrm{SU}_{\mathrm{TX}}-\mathrm{SU}_{\mathrm{RX}}\right)$ and between secondary transmitter and the primary receiver $\left(\mathrm{SU}_{\mathrm{TX}}-\mathrm{PU}_{\mathrm{RX}}\right)$ respectively.

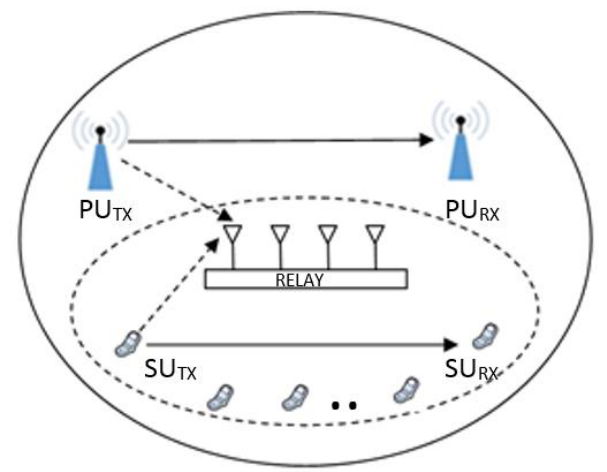

Figure 1 System model

Further let $H$ and $G$ denote the probability-densityfunction (PDF) of the two channels discussed above, and let $h \triangleq\left[h_{1}, h_{2}, \ldots \ldots h_{N}\right]$ and $g \triangleq\left[h_{1}, h_{2}, \ldots \ldots h_{N}\right]$ represents instantaneous channel power gains, all the above parameters are assumed to be known for SUs.

\subsection{0ptimal resource allocation}

This paper examines the resource sharing issues for one user. Let us assume that there are $N$ channels arranged in decreasing order in accordance with their channel-gain to the interference-noise ratio (CINR) quantified at the recipient. The channels sorted so that, assigned channels $K$ can be used to define the start of the channel assignment in place of the binary parameter $x$. B is the bandwidth of each channel, and the PU action indicator is $\omega^{(k)}$ where $k$ is the indicator( 1 to $N)$. This results in the total $K \in$ $\{1, \ldots, N\}$ the number of channels and $p^{(k)} \in \mathcal{R}^{+}\{0\}$ the power turns out to be the user power allocation entities to be found so that the data rate constraint $\phi$ can be fulfilled. The optimization for the resource sharing problem can be articulated as below: $\min _{K, p}\left\{F_{B} F_{P}\right\}: F_{B}=\sum_{k=1}^{K} B \widetilde{\omega}^{(k)}$,

$F_{P}=\sum_{k=1}^{K} p^{(k)}$, provided

$\sum_{k=1}^{K} B \log \left(1+\frac{g^{(k)} p^{(k)}}{\eta+\sum_{p \in P} \mathcal{J}_{p}^{(k)}}\right) \geq \phi$ 
The above problem can be solved in two steps. The process begins by determining the power for the given channels $K^{*}$, and then determines the peak value of $K$ that minimizes the bandwidth-power product on the application of power.

\subsection{Channel allocation}

After locating the optimum power values for a given number of assigned channels $K$, it results in $K$ that minimizes the BP product:

$\min _{K}\left\{\left(\sum_{k=1}^{K} B \widetilde{\omega}^{(k)}\right)\left(\sum_{k=1}^{K} \frac{2 \frac{\phi}{K^{*} B}}{\prod_{K *}\left(h^{(k)}\right)^{1 / K^{*}}}-\frac{1}{h^{(k)}}\right)\right\}$

Gauss-Newton technique [19] can be applied iteratively to solve the above problem. If it is assumed that $\bar{h}$ is the mean $h^{(k)}$ over all channels $k \in$ $\{1,2, \ldots K\}$, and $\Delta^{(k)}$ is the difference between the instant value of CINR $h^{(k)}$ and its mean $\bar{h}$,

This gives $h^{(k)}=\bar{h}+\Delta^{(k)}$. As a result, Equation 3 becomes:

$\min _{K}\left(\sum_{k=1}^{K} \widetilde{\omega}^{(k)}\right)\left(\frac{K 2 \frac{\phi}{K B}}{\left.\prod_{k} \overline{(h}+\Delta^{(k)}\right)^{\frac{1}{K}}}-\sum_{k=1}^{K} \frac{1}{\overline{(h}+\Delta^{(k)}}\right)$

for multiple user

$\min \sum_{j=1}^{|J|} \sum_{k=1}^{K}\left(\frac{B}{1-\omega^{(k)}} x_{j}^{(k)}\right)$

subject to

$\sum_{k \in \overline{\mathcal{K}_{j}}} x_{j}^{(k)}=0, \forall x_{j}^{(k)} \in\{0,1\}$

$\sum_{j=1}^{\left|J_{i}\right|} x_{j}^{(k)} \leq 1, \forall i \in C$

$\sum_{k=1}^{K} B x_{j}^{(k)} \log \left(1+\frac{g_{j}^{(k)} p_{j}^{*(k)}}{\eta+\sum_{p \in P} \mathcal{J}_{p j}^{(k)}}\right) \geq \phi_{j}$.

\subsection{0ptimal power allocation}

2.4.1Ergodic capacity:

For fading channels, ergodic capacity is defined as the maximum attainable rate averaged on all the fading slabs. The solution to the following optimization problem gives peak power assignment.

Pl: $\max _{p 1, p 2} E\left[\sum_{i=1}^{2} r_{i}\right]$,

That gives,

$p_{1} \geq 0, p_{2} \geq 0$,

$g_{1} p_{1}+g_{2} p_{2} \leq I$,
Where $E[$.$] represents the statistical probability$ above all the concerned fading channel gains. This makes it easy to prove that $\mathrm{P}_{1}$ is a convexoptimization problem. Thus, the duality gap is zero and solving its dual problem is the same as solving the main problem. The Lagrangian of this problem can be given as

$L(p, \lambda, \gamma)=E\left[\ln \left(1+h_{1} p_{1}\right)+\ln \left(1+h_{2} p_{2}\right)\right]+$

$\lambda_{1} p_{1}+\lambda_{2} p_{2}-\gamma\left(g_{1} p_{1}+g_{2} p_{2}-I\right)$.

Here $\lambda$ and $\gamma$ are the positive dual variables related to the constrictions. The dual function is $q(\lambda, \gamma)=$ $\max _{p} L(p, \lambda, \gamma)$. The Lagrange dual problem is then given by:

$\min _{\lambda \geq 0, \gamma \geq 0} q(\lambda, \gamma)$

Hence, the optimal solution requires satisfying the Karush-Kuhn-Tucker (KKT) constraints below:

$p_{i} \geq 0, \lambda_{i} \geq 0, \gamma \geq 0$,

$g_{1} p_{1}+g_{2} p_{2} \leq I$,

$\lambda_{i} p_{i}=0$,

$\gamma\left(g_{1} p_{1}+g_{2} p_{2}-I\right)=0$

$\frac{\partial L(p, \lambda, \beta, \gamma)}{\partial p_{i}}=\frac{h_{i}}{1+h_{i} p_{i}}+\lambda_{i}+\gamma g_{i}=0$.

Solving the above constraints using methods as in [6], the optimal solution is then deduced as

$p_{i}^{*}=\left\{\begin{array}{cl}\frac{1}{\gamma g_{i}} \frac{1}{h_{i}}, & \text { if } \frac{1}{\gamma}>\frac{g_{i}}{h_{i}}, \\ 0, & \text { if } \frac{1}{\gamma}<\frac{g_{i}}{h_{i}},\end{array} \forall i \in\{1,2\}\right.$

Where $\gamma$ is determined by solving

$g_{1} p_{1}^{*}+g_{2} p_{2}^{*}=I$.

Then we examined that how interferencetransmission-ratio (ITR) (the ratio of transmission to interference power) influences the transmission strategy of each transceiver. The analysis with three regions is summarized as follows:

$p_{1}^{*}=$

$\left\{\begin{array}{cl}0 & , R_{1}>R_{2}+I \\ \frac{1}{2}\left(\frac{I}{g_{1}}-\frac{1}{h_{1}}+\frac{R_{2}}{g_{1}}\right) & , R_{2}+I>R_{1}>R_{2}-I \\ \frac{I}{g_{1}} & , R_{1}<R_{2}-I\end{array}\right.$

$p_{2}^{*}=$

$\left\{\begin{array}{cl}\frac{I}{g_{2}} & , R_{1}>R_{2}+I \\ \frac{1}{2}\left(\frac{I}{g_{2}}-\frac{1}{h_{2}}+\frac{R_{1}}{g_{2}}\right) & , R_{2}+I>R_{1}>R_{2}-I \\ 0 & , R_{1}<R_{2}-I\end{array}\right.$ 
Combining the results obtained under all the cases, the optimal solution strategy for $\mathrm{Pl}$ can be summarized above.

It is observed that for a transceiver the interference power constraint (IPC) and ITRs define the optimal transmission strategies. Usually, the transceiver with smaller ITR has the opportunity to transmit.

\subsection{0ptimal power allocation in Rician fading channels}

2.5.1Rician fading

A Rician fading channel can be defined by two parameters: $\mathrm{K}$ and $\Omega$. K is the ratio of the power in the direct path to that in other directions. $\Omega$ is the total power $\left(\Omega=v^{2}+2 \sigma^{2}\right)$, The received signal is Rice distributed with parameters

$v^{2}=\frac{K}{1+K} \Omega$ and $\sigma^{2}=\frac{\Omega}{2(1+K)}$

The PDF is given as:

$H(x)=$

$\frac{2(K+1) x}{\Omega} \exp \left(-K-\frac{(K+1) x^{2}}{\Omega}\right) I_{0}\left(2 \sqrt{\frac{K(K+1)}{\Omega}} x\right)$

Where $I_{0}(\cdot)$ is the $0^{\text {th }}$ order revised Bessel function

The CDF $F(n)=1-Q_{1}\left(\frac{v}{\sigma_{n}}, \frac{n}{\sigma_{n}}\right)$

Where $Q_{l}$ (.,.) is the Marcum- $Q$ function. With $\mathrm{H}$ and $\mathrm{G}$ are joint probability-density-function (PDF) of the Rician channel gains

From classical water-filling power assignment problem and its optimal solution as in [19].

$\max _{\left\{p_{i}(h, g), \forall h, g\right\} \in \mathcal{F}^{\prime}} \mathrm{E}_{\mathrm{H}, \mathrm{G}}\left\{H\left(\left\{p_{i}(H, G)\right\}\right)\right\}$

\section{A.Optimal power allocation over peak-transmit- power(PTP) and PIP Conditions:}

In this case, $\mathcal{F}^{\prime}=\{\mathrm{PTP}, \mathrm{PIP}\}$ and the optimal value of Equation 29 is denoted as the maximum of $E_{H, G}\left\{f_{1}(H, G)\right\}$ with $f_{1}(h, g)$ for specific realizations $h$ and $g$ given by

$f_{1}(h, g) \triangleq \max _{p_{i}(h, g)} H\left(\left\{p_{i}(h, g)\right\}\right)$

s.t. $p_{i}(h, g) \leq P_{i}^{p k}, \forall i$

$\sum_{i=1}^{N} g_{i} p_{i}(h, g) \leq Q^{p k}$.

If the optimal solution of the problem in Equation 22-24 is $\left\{p_{i}^{*}\right\}$ then there exists $k, 1 \leq k \leq N$, such that

$p_{s_{i}}^{*}=P_{s_{i}}^{p k}, \forall i, 1 \leq i \leq k-1,0$

$<p_{s_{k}}^{*} \leq P_{s_{k}}^{p k}$, and $p_{s_{i}}^{*}=0, \forall i, k+1 \leq i \leq N$.

This gives the structure of the optimal solution for power allocation over the PTP and PIP conditions.

\section{B. Optimal Power Allocation over Average- Transmit-Power (ATP) and PIP Constraints:} Here,

$\mathcal{F}^{\prime}=\{$ ATP, PIP $\}$ and the dual function is given as:

$f_{4}\left(\left\{\lambda_{i}\right\}\right) \triangleq E_{H, G}\left\{f_{4}^{\prime}(H, G)\right\}+\sum_{i=1}^{N} \lambda_{i} P_{i}^{a v}$

Where $\left\{\lambda_{i} \mid 1 \leq i \leq N\right\}$ are the positive dual variables associated with the ATP conditions. Here $f_{4}^{\prime}(h, g)$ is written as

$f_{4}^{\prime}(h, g) \triangleq \max H\left(\left\{p_{i}(h, g)\right\}\right)-\sum_{i=1}^{N} \lambda_{i} p_{i}(h, g)$

s.t. $\sum_{i=1}^{N} g_{i} p_{i}(h, g) \leq Q^{p k}$.

Denoting the optimal solution of the problem in Equation 28-29 as $\left\{p_{i}^{*}\right\}$, thus, for the optimal power allocation under the ATP and PIP constraints, there exists at most only two users that transmit at nonzero power.

2.5.2Nakagami-m fading

PDF equation as shown below:

$f(x ; m, \Omega)=\frac{2 m^{m}}{\Gamma(m) \Omega^{m}} x^{2 m-1} \exp \left(-\frac{m}{\Omega} x^{2}\right), \forall x \geq 0$.

$m \geq 1 / 2$, and $\Omega>0$ )

Its cumulative distribution function is

$F(x ; m, \Omega)=P\left(m, \frac{m}{\Omega} x^{2}\right)$

The parameters $m$ and $\Omega$ are

$m=\frac{E^{2}\left[X^{2}\right]}{\operatorname{Var}\left[X^{2}\right]}, \quad \Omega=E\left[X^{2}\right]$

\section{Optimal Power Allocation under PTP and PIP} Constraints:

Similar analysis as in A.1 can be performed to find the optimal solution as $\left\{\mathrm{p}_{\mathrm{i}}^{*}\right\}$ then there exists $k, 1 \leq k$ $\leq N$,

such that

$p_{s_{i}}^{*}=P_{s_{i}}^{p k}, \forall i, 1 \leq i \leq k-1,0<p_{s_{k}}^{*} \leq P_{s_{k}}^{p k}$,

and $p_{s_{i}}^{*}=0, \forall i, k+1 \leq i \leq N$.

This gives the structure of the optimal solution for power allocation under the PTP and PIP constraints.

Optimal power allocation under ATP and PIP constraints:

Similar analysis as shown above can be performed to find the optimal solution as $\left\{\mathrm{p}_{\mathrm{i}}^{*}\right\}$,

\section{Results}

This paper considers a cognitive radio network with one PU and a network of SUs. Two separate fading channels the Rician and Nakagami-m are considered. About 1000 randomly generated sets of channel 
power gains $\mathrm{h}$ and $\mathrm{g}$ are used. Other parameters assumed are $\mathrm{W}=1, \sigma^{2}=1$.

Figure 2 shows the simulation of the sum ergodic capacity in PTP+PIP, and PTP+AIP constriction against $P^{p k}$. The simulation is carried out for $\mathrm{N}=2$ and $\mathrm{N}=4$ for Rician fading channel. It can be seen from the figure that the maximum sum ergodic capacity attained in PTP+AIP is higher than that obtained over PTP+PIP for any given number of SUs. This is for the reason that the power assignment is handier for SUs over the ATP constriction than over the PTP constriction. Further achieved maximum ergodic-capacity improves with more number of SUs.

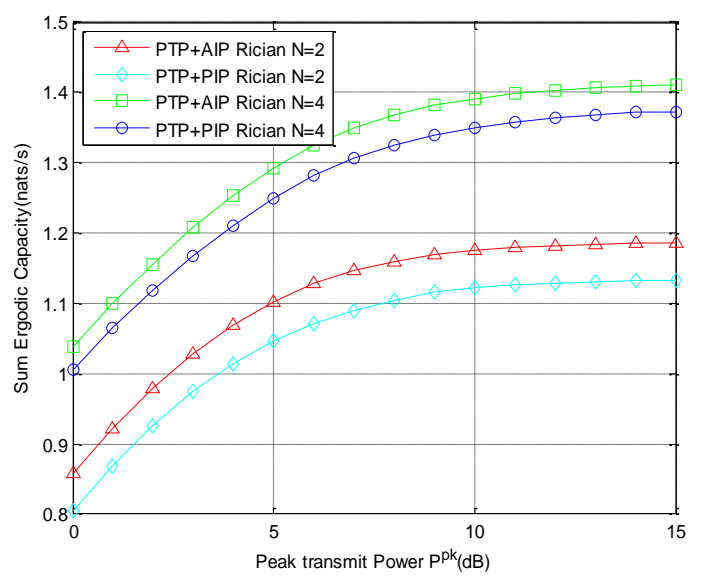

Figure 2 Sum ergodic capacity with Rician fading channel versus $\mathrm{P}^{\mathrm{pk}}$

Figure 3 shows the simulation of sum ergodic capacity in PTP+PIP, and PTP+AIP constriction against $P^{p k}$ with Nakagami-m fading channel for $\mathrm{N}=2$ and $\mathrm{N}=4$. The results are more or less similar as in Figure 2. However, Nakagami-m channel has better maximum sum ergodic capacity than the Rician channel. Further, it can be observed that the performance of ergodic capacity increases with the number of SUs.

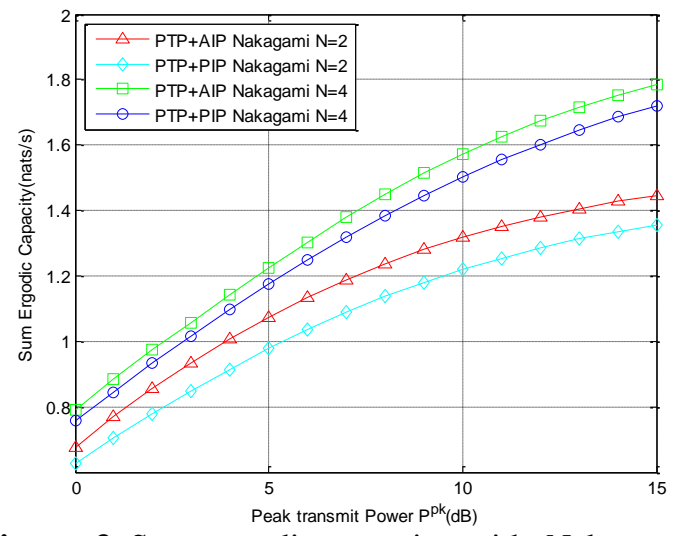

Figure 3 Sum ergodic capacity with Nakagami-m fading channel versus $\mathrm{P}^{\mathrm{pk}}$.

Figure 4 shows the plot of sum ergodic capacity in $\mathrm{PTP}+\mathrm{PIP}$ and ATP+PIP constriction against $Q^{p k}$. The simulation is carried out for $\mathrm{N}=2$ and $\mathrm{N}=4$ for the Rician fading channel. It can be seen from the figure that the maximum sum ergodic capacity attained in ATP+PIP is higher than that obtained under PTP+PIP for any given number of SUs. This is for the reason that the power assignment is handier for SUs over the ATP constriction than over the PTP constriction. Further achieved maximum ergodic-capacity is better with higher number of SUs.

Figure 5 shows the plot of sum ergodic capacity in $\mathrm{PTP}+\mathrm{PIP}$ and ATP+PIP constrictions against $Q^{p k}$. The simulation is carried out for $\mathrm{N}=2$ and $\mathrm{N}=4$ for Nakagami-m fading channel. It can be seen from the figure that the maximum sum ergodic capacity attained in ATP+PIP is higher than that obtained under PTP+PIP for any given number of SUs. This is for the reason that the power assignment is handier for SUs over the ATP constriction than over the PTP constriction. Further achieved maximum ergodiccapacity is better a greater number of SUs. It can also be observed that the Nakagami-m channel has better maximum sum ergodic capacity than Rician channel. 


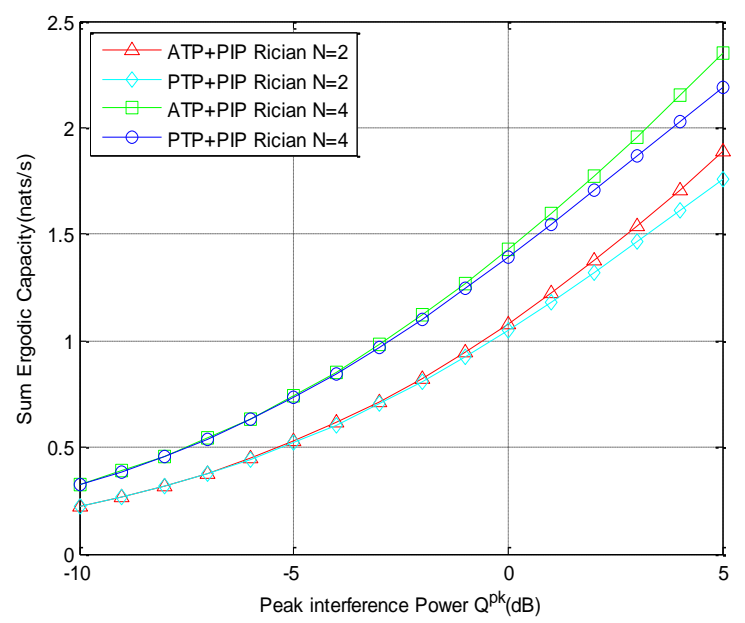

Figure 4 Sum ergodic capacity with Rician fading channel versus $Q^{\text {pk }}$

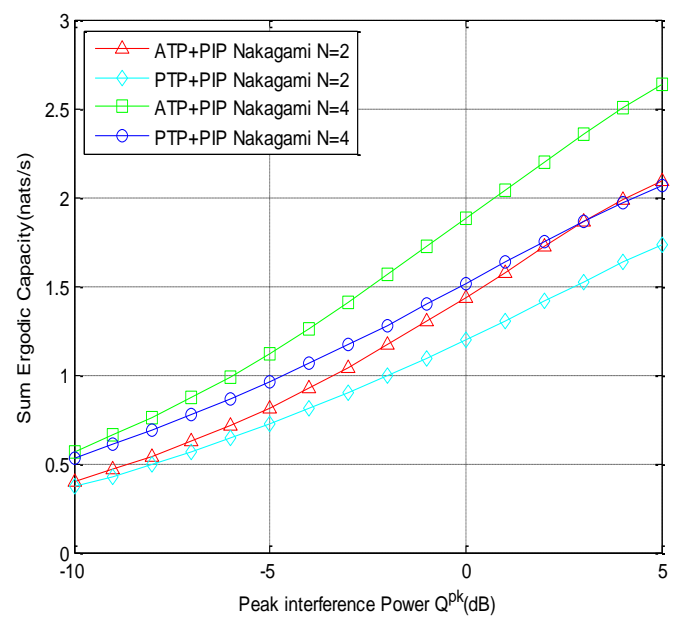

Figure 5 Sum ergodic capacity with Nakagami-m fading channel versus $\mathrm{Q}^{\mathrm{pk}}$

Figure 6 shows the plot of maximum sum ergodic capacity in PTP+AIP, ATP+PIP, ATP+AIP versus W for $\mathrm{N}=2$ and $\mathrm{N}=4$ with Rician fading channel. It can be seen that the increase in the number of SUs results in better maximum sum ergodic capacity. Here it can be observed that with the increase in $\mathrm{W}$ does not cause saturation in the sum ergodic capacities.

Figure 7 shows the plot of maximum sum ergodic capacity in PTP+AIP, ATP+PIP, ATP+AIP versus spectrum(W) for $\mathrm{N}=2$ and $\mathrm{N}=4$ with Nakagami-m fading channel. It can be observed that the performance remains similar to the earlier plots of Nakagami-m fading channel. Again Nakagami-m channel has better maximum sum ergodic capacity than the Rician channel

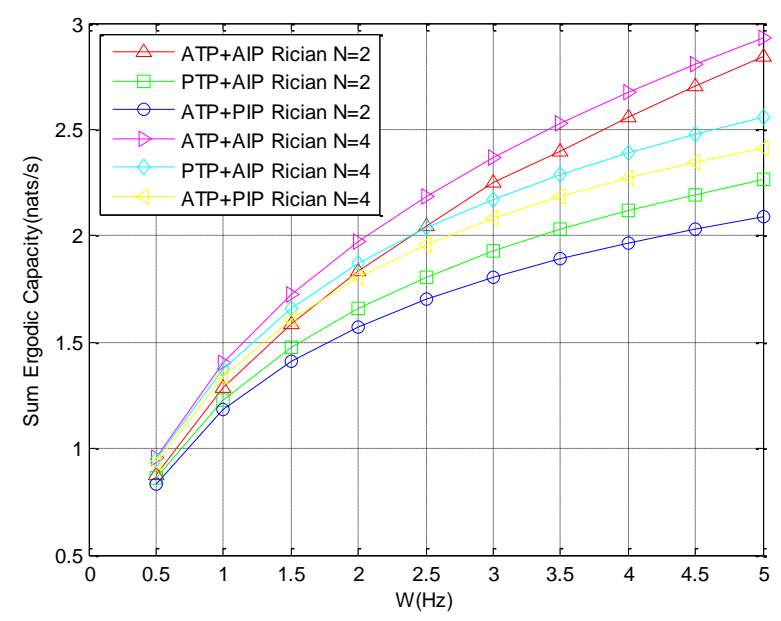

Figure 6 Sum ergodic capacity with Rician fading channel versus $\mathrm{W}$

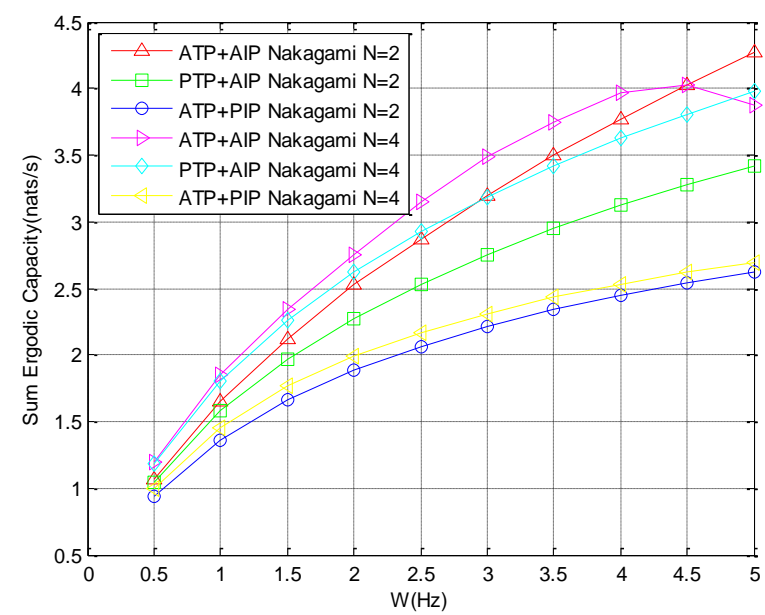

Figure 7 Sum ergodic capacity with Nakagami-m fading channel versus $\mathrm{W}$

\section{Discussion}

This paper emphasizes on the optimal power allocation in Rician and Nakagami channel fading models in CRNs, where multiple SUs share the licensed spectrum of a PU. The sum ergodic capacity of the SUs, which is a relevant network performance metric for delay-tolerant traffics, is studied.

At first, a framework has been developed for the optimal bandwidth and power assignment for a generalized situation and for a generalized channel fading, the solution for this problem is then extended to the problem associated with Rician and Nakagami channel fading states. Closed-form expressions were developed for both scenarios. Besides the peak/average transmit-power constraints at the SUs and the peak/average interference-power constraint 
imposed by the PU. The expressions of peak power allocations are also derived under peak-power and peak-interference conditions.

Most of the literature found deals with optimization of the power and bandwidth allocation in Rayleigh fading model while the work concerns to Rician or Nakagami fading models can be hardly found. In this paper the work has been exclusively carried out for Rician and Nakagami fading models. Further the outcomes of Rician and Nakagami bandwidth and power allocation optimization under different number of SUs are compared.

With respect to Table 1 and Table 2 it can be seen that the Nakagami-m channel has better maximumsum-ergodic capacity than that of the Rician channel. The above result justifies the fact that the slope of the ergodic probability in Nakagami fading is steeper than that in Rician fading. Further, it can be observed that the performance of ergodic capacity increases with the number of SUs.

Table 1 Sum ergodic capacity versus PTP of Rician and Nakagami-m fading channels

\begin{tabular}{lllll}
\hline $\begin{array}{l}\text { PTP } \\
(\text { dB })\end{array}$ & $\begin{array}{l}\text { Rician } \\
\text { Sum-ergodic-capacity }\end{array}$ & & $\begin{array}{l}\text { Nakagami-m } \\
\text { Sum-ergodic-capacity }\end{array}$ \\
\cline { 2 - 5 } & $\mathrm{N}=2$ & $\mathrm{~N}=4$ & $\mathrm{~N}=2$ & $\mathrm{~N}=4$ \\
\hline 0.0 & 0.862 & 1.143 & 0.68 & 0.79 \\
\hline 2.5 & 1.12 & 1.19 & 0.88 & 1.03 \\
\hline 5.0 & 1.11 & 1.286 & 1.81 & 1.23 \\
\hline 7.5 & 1.163 & 1.361 & 1.21 & 1.42 \\
\hline 10.0 & 1.173 & 1.383 & 1.33 & 1.57 \\
\hline 12.5 & 1.181 & 1.40 & 1.41 & 1.72 \\
\hline 15.0 & 1.183 & 1.413 & 1.45 & 1.78 \\
\hline
\end{tabular}

Table 2 Sum ergodic capacity versus PIP of Rician and Nakagami-m fading channels

\begin{tabular}{lllll}
\hline $\begin{array}{l}\text { PIP } \\
(\text { dB })\end{array}$ & $\begin{array}{l}\text { Rician } \\
\text { Sum-ergodic-capacity }\end{array}$ & & \multicolumn{2}{l}{$\begin{array}{l}\text { Nakagami-m } \\
\text { Sum-ergodic-capacity }\end{array}$} \\
\cline { 2 - 5 } & $\mathrm{N}=2$ & $\mathrm{~N}=4$ & $\mathrm{~N}=2$ & $\mathrm{~N}=4$ \\
\hline-10.0 & 0.24 & 0.36 & 0.43 & 0.58 \\
\hline-7.50 & 0.28 & 0.51 & 0.65 & 0.75 \\
\hline-5.0 & 0.53 & 0.73 & 0.78 & 1.13 \\
\hline-2.50 & 0.71 & 1.08 & 1.11 & 1.51 \\
\hline 0.0 & 1.09 & 1.46 & 1.45 & 2.32 \\
\hline 2.5 & 1.51 & 1.89 & 1.83 & 2.69 \\
\hline 5.0 & 1.91 & 2.37 & 2.13 & \\
\hline
\end{tabular}

It can be seen from the Figure 4 and Figure 5 that the maximum sum ergodic capacity attained in ATP+PIP is higher than that obtained under PTP+PIP for any given number of SUs. This is for the reason that the power assignment is handier for SUs over the ATP constriction than over the PTP constriction.

From Figure 6 and Figure 7(also Table 1 and Table 2 ), it is clear that the increase in the number of SUs results in better maximum sum ergodic capacity. Further it can be observed the increase in $\mathrm{W}$ does not cause saturation in the sum ergodic capacities. Again Nakagami-m channel has better maximum sum ergodic capacity than the Rician channel.

The work carried out clearly comes out with a strategy for optimized power allocation for Rician and Nakagami fading models in CRNs which were 130 the main objective of this paper. The investigation and analysis on optimal power and bandwidth allocation can be used for future reference of resource allocation in the CRNs over Rician and Nakagami-m fading channels.

\section{Conclusion}

The radio resource allocation problem in CRNs, where SUs use the spectrum allocated to PUs has been considered. The sum ergodic capacity of all the SUs is taken as the performance metric of the network. While the literature discussed above focuses only on Rayleigh fading channel, the work carried out formulates the optimal power allocation strategies to achieve the fundamental capacity limits of a secondary network with Rician and Nakagami-m fading channels which is the main objective of this paper. In particular, the ergodic capacity is 
considered. Closed-form solutions are obtained for both scenarios. Further the system model considers a network of SUs which is hardly addressed in previous literature. Different peak/average transmitter power constrictions at the SUs and the peak/average interference power constrictions set by the PU are considered. The equations of optimal power allocations are also derived under peak power and peak interference constraints. Simulation results depicted by both figures and tables show that the optimal power and bandwidth allocation for Rician and Nakagami-m fading channels.

\section{Acknowledgment}

None.

\section{Conflicts of interest}

The authors have no conflicts of interest to declare.

\section{References}

[1] Kolodzy P, Avoidance I. Spectrum policy task force. Federal Commun. Comm., Washington, DC, Rep. ET Docket. 2002; 40(4):147-58.

[2] Mitola J, Maguire GQ. Cognitive radio: making software radios more personal. IEEE Personal Communications. 1999; 6(4):13-8.

[3] Haykin S. Cognitive radio: brain-empowered wireless communications. IEEE Journal on Selected Areas in Communications. 2005; 23(2):201-20.

[4] Ghasemi A, Sousa ES. Fundamental limits of spectrum-sharing in fading environments. IEEE Transactions on Wireless Communications. 2007; 6(2):649-58.

[5] Musavian L, Aïssa S. Capacity and power allocation for spectrum-sharing communications in fading channels. IEEE Transactions on Wireless Communications. 2009; 8(1):148-56.

[6] Kang X, Liang YC, Nallanathan A, Garg HK, Zhang R. Optimal power allocation for fading channels in cognitive radio networks: ergodic capacity and outage capacity. IEEE Transactions on Wireless Communications. 2009; 8(2):940-50.

[7] Tian F, Yuan X, Hou T, Lou W, Yang Z. Cost minimization for cooperative traffic relaying between primary and secondary networks. IEEE Transactions on Mobile Computing. 2018; 17(9):2014-27.

[8] Li X, Liu J, Yan L, Han S, Guan X. Relay selection for underwater acoustic sensor networks: a multi-user multi-armed bandit formulation. IEEE Access. 2018; 6:7839-53.

[9] Singh K, Gupta A, Ratnarajah T, Ku ML. A general approach toward green resource allocation in relayassisted multiuser communication networks. IEEE Transactions on Wireless Communications. 2018; 17(2):848-62.

[10] Feng Y, Leung VC, Ji F. Performance study for SWIPT cooperative communication systems in shadowed nakagami fading channels. IEEE Transactions on Wireless Communications. 2018; 17(2):1199-211.

[11] Jitvanichphaibool K, Zhang R, Liang YC. Optimal resource allocation for two-way relay-assisted OFDMA. IEEE Transactions on Vehicular Technology. 2009; 58(7):3311-21.

[12] Sidhu GA, Gao F, Chen W, Nallanathan A. A joint resource allocation scheme for multiuser two-way relay networks. IEEE Transactions on Communications. 2011; 59(11):2970-5.

[13] Li H. A recommendation system in cognitive radio networks with random data traffic. IEEE Transactions on Vehicular Technology. 2011; 60(4):1352-64.

[14] Kumar NS, Vakula D. Performance analysis of equalizers for MIMO in Rician-K and Nakagami-m fading channels. In international conference on electronics, communication and aerospace technology 2017(pp. 381-5). IEEE.

[15] Sharma PK, Priya VH, Raju GK, Sai KV. Comparison of MIMO over different fading channels with and without channel state information. In second international conference on electronics, communication and aerospace technology 2018 (pp. 730-3). IEEE.

[16] Ujjinimatad R, Patil SR. Spectrum sensing in cognitive radio networks with known and unknown noise levels. IET Communications. 2013; 7(15):170814.

[17] Ahmed JF, Ujjinimatad R. Energy detection with different digital modulation techniques over Rayleigh fading channels in cognitive radio networks. In innovations in power and advanced computing technologies 2017 (pp. 1-5). IEEE.

[18] Fareduddin AJ, Ujjinimatad R. Fast optimal and explorative (FOX) sensing and power allocation scheme for non-cooperative cognitive radio networks. In international conference on recent trends in electronics, information \& communication technology 2017 (pp. 457-61). IEEE.

[19] Bertsekas DP. Nonlinear programming. Belmont: Athena Scientific; 1999. 


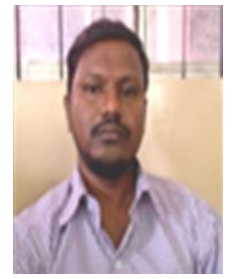

Fareduddin Ahmed J S received B. E degree in Electronics and Communication Engineering from Gulbarga University and M. Tech in Computer Networks Engineering from VTU, Belgaum. $\mathrm{He}$ is currently pursuing Ph.D in Electrical and Electronics Engineering from VTU, Belgaum. He has a teaching experience of 17 years and worked as HOD in ECE Department. Currently, he is working as Assistant Professor in ECE Department at BITM Bellary, India.

Email: fareduddin@gmail.com

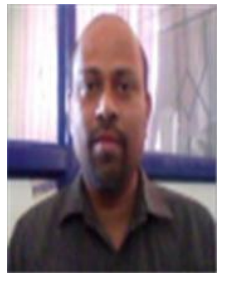

Rohitha Ujjinimatad received B. E degree in Electronics and Communication Engineering from Bangalore University, M. Tech in Digital Electronics from VTU, Belgaum and $\mathrm{Ph} . \mathrm{D}$ degree in Electrical and Electronics Engineering from VTU, Belgaum in 2015. He is a member of IEEE and life member of ISTE. Currently, he is working as a Professor and HOD in ECE Department at PDIT Hospet, India.

Email: rohitha_ujjini@rediffmail.com 